\title{
JetGene-Online Database and Toolkit for an Analysis of Regulatory Regions or Nucleotide Contexts at Differently Translated Plants Transcripts
}

\author{
Nataliya Sadovskaya ${ }^{1, *}$, Orkhan Mustafaev ${ }^{2}$, Alexander Tyurin ${ }^{1}$, Igor Deyneko ${ }^{1}$ and Irina \\ Goldenkova-Pavlova ${ }^{1}$ \\ 1 Timiryazev Institute of Plant Physiology, Russian Academy of Sciences, ul. Botanicheskaya 35, \\ 127276 Moscow, Russia \\ 2 Genetic Resources Institute, Azerbaijan National Academy of Sciences, Azadlig ave. 155, \\ AZ1106 Baku, Azerbaijan; orkhan@bioset.org \\ * Correspondence: nataliya.sadovskaya@gmail.com; Tel.: +07-499-678-5400 \\ + The 1st International Electronic Conference on Plant Science, 1-15 December 2020. \\ Published: 30 November 2020
}

\begin{abstract}
RNAs has some regulatory codes which can define the fate of an individual mRNA in translation. We have developed a flexible online database JetGene (https://jetgene.bioset.org/) that contains cDNA, CDS, 5'-UTR, 3'-UTR sequences from Bacteria, Fungi, Metazoa, Plants, Protists and Vertebrates with the aim of regulatory codes searching in mRNA and studying their correlation with a translational efficiency. It has a friendly interface and puts together a set of tools which are necessary for designing experiments. JetGene allows to do a benchmark analysis of sequences, namely: (1) to estimate the variation of length, nucleotide composition, frequency of codon usage, to analyze GC-content, CpG-islands, to study nucleotides surrounding of the start codon and much more; (2) to identify and define statistically significant representation of potential regulatory contexts at mRNA with different translation efficiency. A user can make a bioinformatics analysis for full-length transcripts or for a fragment of transcripts or for coding/non-coding regions. Every step of the work is accompanied by graphical interpretation of results. Moreover, beta-version of JetGene (https://beta.bioset.org, under construction) allows user to compare two datasets of mRNA and to apply omics data for searching and prediction regulatory determinants of translation.
\end{abstract}

Keywords: in silico analysis; regulatory codes; motives; translation effectivity; comparison two datasets of mRNA

\section{Introduction}

Translation is a fundamental process and an important starting point in gene expression regulation for cells of all living organisms because in this process encoding potential of mRNA is exposed via the protein molecule. In the current view, translational control in general is decisive in the continuity of cell events and, for example, in response of plant cells to various environmental factors and different metabolites [1]. The special attention of researchers is focused at discrepancy between mRNAs levels and translation effectivity in eukaryotic cells, in particular in plant cells [2,3]. The experimental data of the various elegant studies show that when decoding their genomes, organisms are able to widely use the regulation and decoding rules of higher orders along with the canonical translational rules, thereby suggesting the presence of specific regulatory codes characteristic of the mRNA translation.

As we know, cDNA includes the following parts: 5' untranslated region (5'-UTR), coding region (CDS) and 3' untranslated region (3'-UTR). These regions modulate translation at "control points": initiation, elongation and translation termination. According to the current opinion, numerous regulatory codes could be hidden in nucleotide contexts of such cDNA regions. Each element 
separately or some of them in combination can determine the fate of an individual mRNA in translational process [4]. In silico analysis of cDNA parts, which have mentioned above: CDS, $5^{\prime}$-UTR and 3'-UTR, is applied for prediction of these regulatory codes.

For the purpose of such regulatory codes discovery in mRNA and their correlation with efficiency of translation we have created online database JetGene (https://jetgene.bioset.org/). In addition JetGene allows to estimate the variation of nucleotide composition, codon usage frequency, to study nucleotides surrounding of the start codon and much more.

\section{Experiments}

\subsection{The Motivation for the Development of JetGene}

Our goal of creation JetGene is to provide users that have minimal experience in programming and in a bioinformatics analysis with a simple and useable toolkit for an analysis and planning of an experiment. So in JetGene we have put together a wide set of options which are useful for any researcher. JetGene allows to make a comparative analysis of sequences, such as: (1) to estimate the variation of length, nucleotide composition, frequency of codon usage, to analyze GC-content, CpG-islands, to study nucleotides surrounding of the start codon and much more; (2) to identify and define statistically significant representation of potential regulatory contexts at mRNA with different translation efficiency. JetGene contains cDNA, CDS, 5'-UTR, 3'-UTR sequences for six groups of living organisms: Bacteria, Fungi, Metazoa, Plants, Protists and Vertebrates. It should be noted that the analysis could be performed both on full-length transcripts, and on truncated transcripts and on coding/non-coding regions.

In addition, beta-version of JetGene (https://beta.bioset.org, under construction) allows user to compare two mRNA datasets (Figure 1) and to apply omics data for searching and prediction regulatory determinants of translation.

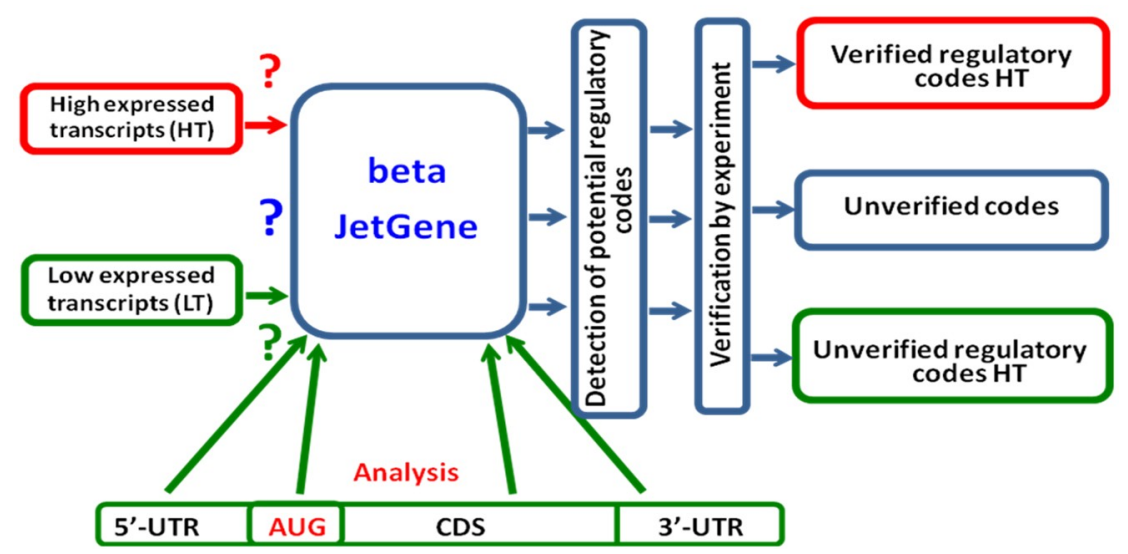

Figure 1. Comparison circuit of two user individual datasets with the aim of detection and verification regulatory codes in high expressed/low expressed transcripts.

\section{2. "System of Nested Datasets" Algorithm}

Another important advantage of JetGene is a "System of nested datasets" algorithm, which we have implemented in our work (Figure 2). Its essence is that at the first stage of work a researcher selects a certain criterion as a primary one, for example (1) cDNA with the specified length "CDNA length", and creates the main dataset. At the subsequent stages a researcher can use remaining parameters as additional ones, for example, (2) add parameter " $5^{\prime}$-UTR length". It will allow to choose sequences with the specified $5^{\prime}$-UTR length and to create the second order dataset. Then a researcher can add the next parameter, for example motive search "Motifs". As a result of such step JetGene will select sequences containing this motif from the second order dataset. 


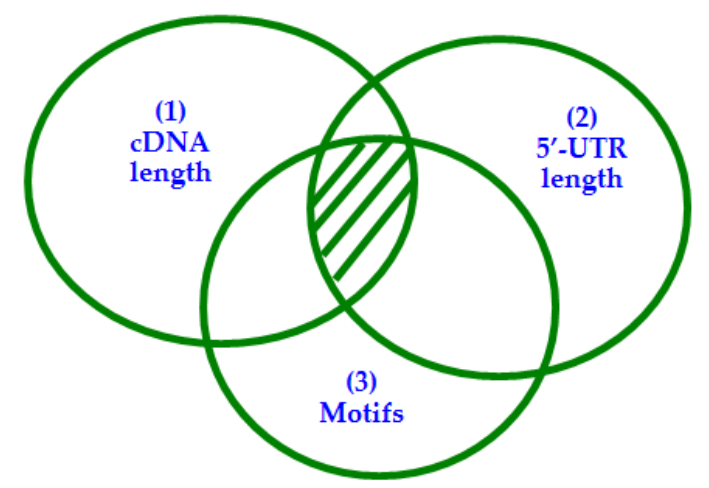

Figure 2. "System of nested datasets" algorithm". Three overlapping circles represent an opportunity to choose sequences by criteria "cDNA length", "5'-UTR length", "Motifs". (1) "cDNA length" is selected as a primary criterion, (2) "5'-UTR length" and (3) "Motifs" - as additional ones. The resulting dataset is located at intersection of all circles and shaded.

So a user has the ability to create a series of subsequent datasets each of which is based on the previous ones without extracting intermediate results from JetGene. A researcher can define criteria hierarchy (main and auxiliary). As a result a user has to obtain different variants of biological texts that satisfy nontrivial parameter combinations. The number of such combinations is unlimited. Besides graphical representation of analysis results is realized in JetGene. All of this greatly simplifies in silico analysis.

\section{Results}

\subsection{Database Overview}

Transcriptomic data of six key groups of living organisms: Bacteria (44048 species), Fungi (782 species), Metazoa (68 species), Plants (45 species), Protists (195 species) and Vertebrates (139 species) were downloaded from Ensembl (https://www.ensembl.org/index.html) [5] on 28 June 2017 and updated regularly (once a week). Description of each transcriptome includes information about assembly. Gene Ontology Annotation (GO) [6] is given for many transcriptomes. The main interface of JetGene contains four major sections: cDNA data, CDS data, 5'-UTR data and 3'-UTR data (Figure 3) for most eukaryotic organisms. It should be noted that we obtain information about $5^{\prime}$-UTR and 3'-UTR as subtraction CDS from cDNA. In addition to the major ones, JetGene has one auxiliary GO-section. It's presented only when information about GO-annotations is provided by Ensembl server and this section is unrelated to the major ones.

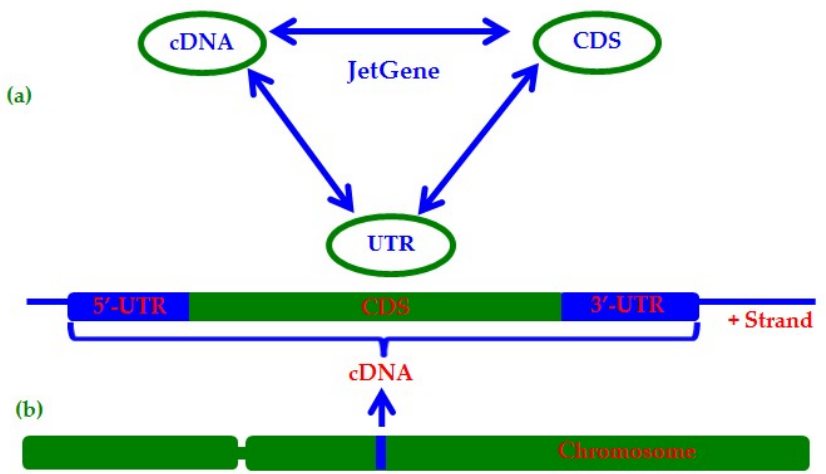

Figure 3. (a) General structure of JetGene. Arrows indicate that a user could start the analysis with any section (CDNA, for example) and continue it at any another section (CDS or UTR, for example) 
without extracting intermediate results from JetGene; (b) Schematic gene representation on a chromosome (introns are removed).

JetGene is implemented in a modular form. Modules could be applied both individually and in combination for conducting extended and continuous research. Web-interface of database consists of 10 main modules inherent to any of four major sections ("CDS", "cDNA", "5'-UTR", "3'-UTR") and three modules inherent to the section "CDS data". The list of modules available for every section is shown at Figure 4.

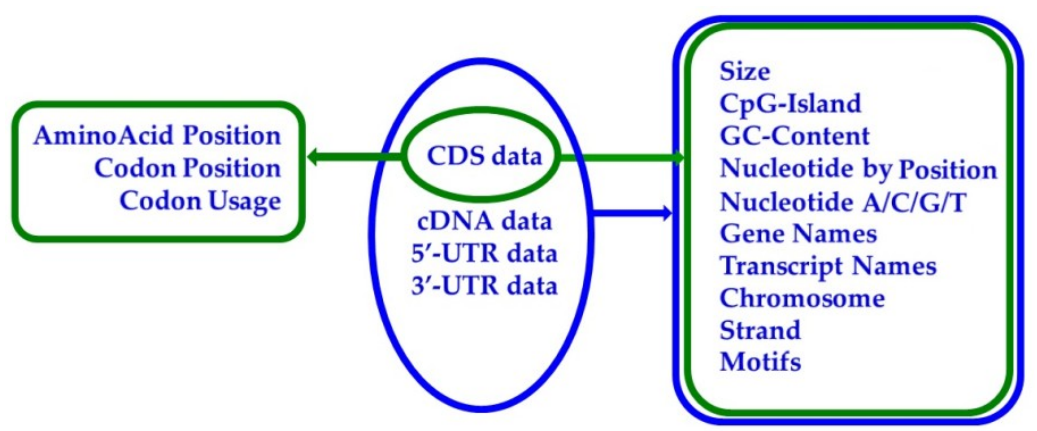

Figure 4. A toolkit of four major sections of JetGene. Tools that accessible for every of the available data types (CDS, CDNA, 5'-UTR, 3'-UTR) are shown schematically.

It's important to note that a user can extract the obtained sequences in fasta-format at any step of the work. Moreover JetGene gives a visual representation for comparison of the performed analysis of the narrow user dataset with an initial transcriptome dataset for researched organism. Besides there is a possibility to upload user dataset and to analyze it (this option is available after free registration). In this case all toolkits will be available except "chromosome", "motifs" and "strand", besides that, a sequence don't markup on CDS, CDNA, 5'-UTR, 3'-UTR.

Here we give a list of modules specific for every section (major and auxiliary).

Modules specific to "CDS data" only:

1. AminoAcid Position

2. Codon Position

3. Codon Usage

Modules specific to "CDS data”, "cDNA data”, “5'-UTR data”, “3'-UTR data”:

1. CDS/cDNA/5'-UTR/3'-UTR Length

2. CpG-Island in CDS/cDNA/5'-UTR/3'-UTR

3. GC-Content in CDS/cDNA/5'-UTR/3'-UTR

4. Nucleotide by Position in CDS/CDNA/5'-UTR/3'-UTR

5. Nucleotide $A / C / G / T$ in $C D S / c D N A / 5^{\prime}-U T R / 3^{\prime}-U T R$

6. Gene Names

7. Transcript Names

8. Chromosome

9. Strand

10. Motifs

Module specific to GO:

1. Gene Ontology Annotations

Further we provide a brief description of modules speciphic for each of four major sections "CDS data", "cDNA data", "5'-UTR data", "3'-UTR data" in details.

\subsection{Modules Specific to "CDS Data" Only}




\subsubsection{AminoAcid Position}

This module makes it possible to display an amino acid that is located on sequence in the position 1-10 both from C-terminus and from N-terminus. It can be helpful for an analysis and designing of signal peptides [7] and for applying the N-end rule. According to this rule the second $\mathrm{N}$-terminal amino acid of a protein determines its half-life [8].

\subsubsection{Codon Position}

This utility is similar to the previous one. It defines which nucleotide triplets are located in the position 1-10 forming 5'-end or 3'-end of CDS. With this application user can study N-terminal region of protein or of signal peptide at the nucleotide level.

\subsubsection{Codon Usage}

Current tool shows triplets encoding amino acids in CDS and also their numerical and percentage composition (we take the sum of all triplets encoding present amino acid as $100 \%$, but we don't take the sum of all triplets in CDS). This tool allows to study full-length CDSs and truncated sequences of CDSs (an option "Sequence region to calculate data (\%)"). Such utility will be helpful for works similar to [9], in which authors analyzed the codon usage of adenoviral proteins and evaluate their adaptation to the host codons.

\subsection{Modules Specific to "CDS Data", "cDNA Data", "5'-UTR Data", "3'-UTR Data"}

\subsubsection{CDS/CDNA/5'-UTR/3'-UTR Length}

This module displays all length of CDS/CDNA/5'-UTR/3'-UTR sequences in transcriptome of the studied organism. It gives a possibility to choose sequences of a certain length (scale division is 500 nucleic acids) or to set a length range at option "Values interval to calculate data". Such utility can be useful for sequences choice with a maximum length for gene cloning into a certain vector.

\subsubsection{CpG-Island in CDS/CDNA/5'-UTR/3'-UTR}

This application analyzes CpG-islands and calculates percent of CpG dinucleotides in CpG-islands in CDS/cDNA/5'-UTR/3'-UTR. The tool allows to choose all sequences with certain percent interval of $\mathrm{CpG}$ dinucleotides in $\mathrm{CpG}$-islands. Moreover it works both with full-length and truncated sequences (an option "Sequence region to calculate data (\%)").

\subsubsection{GC-Content in CDS}

Current tool is similar to the "GpC-island in CDS/CDNA/5'-UTR/3'-UTR" but it takes into account all $G$ and $C$ nucleotides in transcripts. User have an ability to pick up all transcripts that have certain GC-content (scale division is 1\%). This utility can be applied in research similar to [10], in which authors analyzed codon usage in CDSs of H. manillensis and also distribution of GC dinucleotides content in CDSs.

\subsubsection{Nucleotide by Position in CDS/CDNA/5'-UTR/3'-UTR}

This application shows what nucleotide is located in the position 1-10 form 5'-end or from $3^{\prime}$-end of CDS/CDNA/5'-UTR/3'-UTR. It can be useful in works similar to [11], in which authors analyzed immediate upstream region of the $5^{\prime}$-UTR from the AUG start codon in different genes of A. thaliana and showed that a region from positions -1 to -5 is most important for translational efficiency.

\subsubsection{Nucleotide A/C/G/T in CDS/CDNA/5'-UTR/3'-UTR}

This utility can calculate percentage of A/C/G/T in CDS/CDNA/5'-UTR/3'-UTR. It analyzes both full-length and truncated sequences (an option "Sequence region to calculate data $(\%)$ "). Moreover 
user can pick up all sequences with a certain percentage (option "Values interval to calculate data") and to form dataset of sequences with a certain nucleotide composition. Such tool could be useful in works like [4] in which scientists revealed influence of 5'-UTR mono- and di-nucleotide composition on ribosome loading in A. thaliana.

\subsubsection{Gene Names}

This module enables to select sequences by list of names or select sequences that have the common part of their names. Apart from that it allows to upload user dataset by standard gene names if information about an organism is represented in JetGene.

\subsubsection{Transcript Names}

Current application is similar to "Gene names" but user can find unique transcript(s) or all transcripts, related to a certain gene or transcripts that have the common part of names. The utility enables to identify all isoforms of a certain gene easily and to find some difference between them.

\subsubsection{Chromosome}

Current tool shows sequence distribution on chromosomes and on mitochondrial DNA. It can be useful in cases when a researcher is interested in sequences that are located on a certain chromosome or when user compares two datasets obtained for two different chromosomes.

\subsubsection{Strain}

Current utility allows to distinguish transcripts located on forward strand from transcripts located on reverse strand and then to divide dataset at two different parts based on this parameter. For bacteria such simple manipulation makes it possible to find genes that are assignmentid incorrectly to the one operon. Moreover this tool can be useful in research like [12], in which authors showed little asymmetry between forward/reverse strands on open reading frame number and between lengths of genes in C. acetobutylicum.

\subsubsection{Motifs}

This module find out sequences that contain a certain motifs. It can search several motifs simultaneously (by means of an operator AND) or one of listed motifs (by means of an operator OR). User can perform an analysis on full-length sequences and on truncated transcripts. The results are visually presented as a bar graph that displays motif occurrence frequency.

\section{Discussion}

\subsection{Comparison JetGene with Other Online Databases}

We have created JetGene that is accessible via the web interface and very simple in use. It is developed not for experienced bioinformatics only but for experimentalists, who have minimal experience in a bioinformatics analysis and in programming. Let's compare JetGene with other online databases.

Currently biological texts of sequences are stored in different web servers. Most frequently such recourses contain CDSs and protein sequences corresponding to them, as for example in GenBank [13] and in KEGG [14,15]. Furthermore they contain metabolic pathways maps, software package Blast $[16,17]$ for searching homologous sequences, list of publications, links to external Internet resources which provide a comprehensive description of the studied gene or protein and much more. Notwithstanding diversity of represented information, when user works with such databases the search is possible at a trivial level only: find a sequence with a given function or to detect a homologous sequence. 
Than we should describe web resources that allow to conduct a complex analysis of sequences. These include Ensembl (https://www.ensembl.org/) [5], which served as the basis for JetGene. It should be mentioned that JetGene contains information about all organisms and about all nucleotide sequences represented in Ensembl. Nowadays Ensembl is one of the most important Internet resources which store information about gene annotation, genetics, comparative genomics and epigenomics for a huge number of living organisms. Possibilities of using Ensembl range from a quick overview of information to whole-genome in silico analysis. Meanwhile Ensembl support access via BioMart [18] via Perl and REST APIs $[19,20]$ or via FTP for providing access to information the user is interested in. However BioMart don't use whole information that is stored in Ensembl. For example, BioMart does not use information about many organisms represented in Ensembl. Besides using API и FTP requires programming skills that not all users have.

However, BioMart provides an opportunity to work separately with CDS, CDNA, 5'-UTR, $3^{\prime}$-UTR and with protein sequences. Biomart toolkit is larger than JetGene toolkit. In particular BioMart allows to set chromosome coordinates, to obtain information about intron-exon structure, to do a search by phenotype, to find orthologous in other organisms and much more. Herewith the intersection between BioMart toolkit and JetGene toolkit is insignificant. Particularly both BioMart and JetGene give the opportunity to display CDS, CDNA, 5'-UTR, 3'-UTR sequences, to find a gene by ID or some genes by GO (gene ontology annotation), to choose chromosome for an analysis. Nevertheless such essential information as sequence length, GC-content, sequence location at forward/reverse strand is displayed in resulting file. So a user should select sequences manually form resulting file by parameters mentioned above and this increases time of an analysis.

In addition some information, for instance, percentage of nucleotide $A / C / G / T$ or what nucleotide located in the position 1-10, the distribution of triplets within the dataset, is not provided by BioMart. The possibility to work with truncated sequences implemented by BioMart is not so clearly as by JetGene. Apart from that graphical representation of analysis results by the selected parameter is omitted.

Moreover there are a number of limitations when user trying to make several iterations of the analysis or when user trying to do transfers between CDNA/CDS/UTR. For example, it's more difficult to begin with 5'-UTR analysis, than to transfer to analysis of cDNA (cDNA which contains researched 5'-UTR) without additional supporting actions.

UCSC Genome Browser (https://genome.ucsc.edu/) [21,22] is another information resource that allows to make a comprehensive search and analysis of sequences. It contains information about more than 100 speciess, for some of them it has several variants of transcriptome assembly. At the same time UCSC Genome Browser covers fewer kingdoms than JetGene. And any kingdom includes less number of organisms than JetGene. For instance it does not contain any information about Plants, besides that, information about Fungi provided for S. cerevisiae only.

UCSC Table Browser is a flexible and powerful graphical interface designed for manipulating and querying UCSC Genome Browser. Table Browser alike JetGene allows to select sequences by several user criteria, to form sequences dataset with help of some tools and extract obtained dataset in fasta-format. Nevertheless UCSC settings are less clear than JetGene settings. In order to be able to form a correct request or to apply multiple query criteria, to download user data and to use information of this internet resource user should study the structure of the input/output data, the description of filters and to have some bioinformatics knowledge. When researcher solve similar tasks regularly it's justified. But learning settings and options regularly takes considerable time when tasks change rapidly or when selection of sequences is based on different criteria. It should be noted that graphical interpretation of results is not realized in UCSC Table Browser.

At the same time data from UCSC Table Browser can be exported directly to open web-based platform Galaxy (http://usegalaxy.org) [23], but it takes additional time. Some options of Galaxy are the same as for JetGene tools (for example, CDS, CDNA, 5'-UTR, 3'-UTR analysis, GC-content analysis, an ability to choose sequences by length, an opportunity to study both full-length and truncated sequences, a possibility to extract sequences in fasta-format) and graphical visualization of results is implemented in it. Nevertheless, Galaxy options are not so clearly defined as JetGene tools. 
Additionally it should be noted, that both Galaxy and JetGene can do transfers between CDNA/CDS/5'-UTR/3'-UTR. But Galaxy makes such transfers in a less trivial form and they take a longer period of time.

\subsection{Usage of JetGene}

As an example of JetGene usage (it was called FlowGene initially) we can cite the article [24]. In this work authors studied the influence of 5 '-UTR nucleotide context on the gene expression in plants and used JetGene for a bioinformatics analysis. They applied the "System of nested datasets" algorithm. Researchers select the (1) 5'-UTR of not less than two thousand base pair (minimum size of CpG-island) as a primary parameter and created the main dataset. Then they selected additional criteria for creating subsequent datasets: (2) GC-content higher than 50\% (one of the characteristics of CpG-islands); (3) nucleotides surrounding of the start codon at positions +4 and -3 according to Kozak sequence [25]; (4) the absence of alternative start and stop codons within sequences. Then they searched for six-nucleotide motifs, which are contained not less than in $50 \%$ in all sequences from the result dataset. Subsequently these motifs were incorporated into the design of the synthetic sequence.

\section{Conclusions}

Fluctuations in nucleotide composition revealed in genomes of all organisms and they define gene expression efficiency for any species [26-29]. Knowledge about the fine mechanisms of translation is very important for understanding what makes organism to switch genes. Applying information about nucleotide context variations helps to develop antiviral vaccines [30] allows to select host expression system for an experiment [31] to predict genes based on genomic sequences $[32,33]$ to design degenerate primers [34] and much more. Research of fluctuations in nucleotide composition occupies a central position in such important areas as molecular evolution [35] and biotechnology [36]. The availability of genome-wide sequences allows a unique opportunity to identify regularities in the distribution of various properties [37] both across the whole genome and for parts of separate transcript. Thus for example it was identified a dependence between nucleotide composition and efficiency of protein translation [38]. It was established dependence between nucleotide composition and level of gene expression [39,40]. And it was also shown change in nucleotide composition depending on localization of the sequence [41] and much more.

In such studies success is highly dependent on the ability to form sequences datasets of biological texts based on a wide range of criteria. The greater the number of parameters involved in the analysis, the higher the potential for creating and manipulating of sequence datasets. So it will be greater the potential for searching and identifying characteristics which influence on biological properties of sequences. In accordance with all above requirements we have created online database JetGene which allows carry out such analysis quickly and efficiently. It is important to note that currently it gives a comprehensive understanding of the structure-function potential of the biological texts encoded in mRNAs. JetGene is developed for an analysis of nucleotide sequences only and aimed at experimentalists, who have minimal experience in bioinformatics analysis. Uniqueness of our database is that any user able to scan huge amounts of information within shortest time or can create different datasets of nucleotide sequences de novo, which satisfy the goals of the experiment. In this way, a researcher can apply a wide set of options based on different user criteria for conducting a comprehensive analysis, then to form nucleotide sequences dataset and extract it in fasta-format from JetGene. In addition graphical representation of results accompanies every phase of the study. Such cute details are greatly facilitated the work of any user.

Author Contributions: O.M. developed JetGene; N.S., A.T. and I.D. tested JetGene; N.S. and I.G.-P. wrote the paper; I.G.-P. supervised the project. All authors have read and agreed to the published version of the manuscript.

Acknowledgments: The authors gratefully acknowledge financial support from Russian Science Foundation (project no. 18-14-00026; IVG-P). 
Conflicts of Interest: The authors declare no conflict of interest.

\section{Abbreviations}

The following abbreviations are used in this manuscript:

$\begin{array}{ll}\text { CDS } & \text { Coding DNA Sequence } \\ \text { cDNA } & \text { Complementary DNA } \\ \text { GO } & \text { Gene Ontology Annotation } \\ \text { HT } & \text { High Expressed Transcripts } \\ \text { LT } & \text { Low Expressed Transcripts } \\ \text { UTR } & \text { Untranslated Region }\end{array}$

\section{References}

1. Guerra, D.; Crosatti, C.; Khoshro, H.; Mastrangelo, A.M.; Mica, E.; Mazzucotelli, E. Post-transcriptional and post-translational regulations of drought and heat response in plants: A spider's web of mechanisms. Front. Plant Sci. 2015, 6, 57, doi:10.3389/fpls.2015.00057.

2. Bärenfaller, K.; Grossmann, J.; Grobei, M.A.; Hull, R.; Hirsch-Hoffmann, M.; Yalovsky, S.; Zimmermann, P.; Grossniklaus, U.; Gruissem, W.; Baginsky, S. Genome-scale proteomics reveals Arabidopsis thaliana gene models and proteome dynamics. Science 2008, 320, 938-941, doi:10.1126/science.1157956.

3. Bärenfaller, K.; Massonnet, C.; Walsh, S.; Baginsky, S.; Buhlmann, P.; Hennig, L.; Hirsch-Hoffmann, M.; Howell, K.A.; Kahlau, S.; Radziejwoski, A.; et al. Systems-based analysis of Arabidopsis leaf growth reveals adaptation to water deficit. Mol. Syst. Biol. 2012, 8, 606, doi:10.1038/msb.2012.39.

4. Kawaguchi, R.; Bailey-Serres, J. mRNA sequence features that contribute to translational regulation in Arabidopsis. Nucleic Acids Res. 2005, 33, 955-965, doi:10.1093/nar/gki240.

5. Yates, A.; Achuthan, P.; Akann, W.; Allen, J.; Allen, J.; Alvarez-Jarreta, J.; Amode, M.R.; Armean, I.; Azov, A.; Bennett, R.; et al. Ensembl 2020. Nucleic Acids Res. 2020, 48, D682-D688, doi:10.1093/nar/gkz966.

6. Carbon, S.; Douglass, E.; Dunn, N.; Good, B.; Harris, N.L.; Lewis, S.E.; Mungall, C.J.; Basu, S.; Chisholm, R.L.; Dodson, R.J.; et al. The Gene Ontology Resource: 20 years and still GOing strong. Nucleic Acids Res. 2019, 47, D330-D338, doi:10.1093/nar/gky1055.

7. Jeiranikhameneh, M.; Moshiri, F.; Keyhan Falasafi, S.; Zomorodipour, A. Designing Signal Peptides for Efficient Periplasmic Expression of Human Growth Hormone in Escherichia coli. J. Microbiol. Biotechnol. 2017, 27, 1999-2009, doi:10.4014/jmb.1703.03080.

8. Tasaki, T.; Sriram, S.M.; Park, K.S.; Kwon, Y.T. The N-end rule pathway. Annu. Rev. Biochem. 2012, 81, 261-289, doi:10.1146/annurev-biochem-051710-093308.

9. Villanueva, E.; Martí-Solano, M.; Fillat, C. Codon optimization of the adenoviral fiber negatively impacts structural protein expression and viral fitness. Sci. Rep. 2016, 6, 27546, doi:10.1038/srep27546.

10. Guan, D.L.; Ma, L.B.; Khan, M.S.; Zhang, X.X.; Xu, S.Q.; Xie, J.Y. Analysis of codon usage patterns in Hirudinaria manillensis reveals a preference for GC-ending codons caused by dominant selection constraints. BMC Genomics 2018, 19, 542, doi:10.1186/s12864-018-4937-x.

11. Kim, Y.; Lee, G.; Jeon, E.; Sohn, E.J.; Lee, Y.; Kang, H.; Lee, D.W.; Kim, D.H.; Hwang, I. The immediate upstream region of the $5^{\prime}$-UTR from the AUG start codon has a pronounced effect on the translational efficiency in Arabidopsis thaliana. Nucleic Acids Res. 2014, 42, 485-498, doi:10.1093/nar/gkt864.

12. Zhao, H.L.; Xia, Z.K.; Hua, Z.G.; Wei, W. Selectional versus mutational mechanism underlying genomic features of bacterial strand asymmetry: A case study in Clostridium acetobutylicum. Genet. Mol. Res. 2015, 14, 1911-1925, doi:10.4238/2015.

13. Benson, D.; Cavanaugh, M.; Clark, K.; Karsch-Mizrachi, I.; Lipman, D.; Ostell, J.; Sayers, E. GenBank. Nucleic Acids Res. 2017, 45, D37-D42, doi:10.1093/nar/gkw1070.

14. Kanehisa, M.; Goto, S.; Sato, Y.; Kawashima, M.; Furumichi, M.; Tanabe, M. Data, information, knowledge and principle: Back to metabolism in KEGG. Nucleic Acids Res. 2014, 42, D199-205, doi:10.1093/nar/gkt1076.

15. Kanehisa, M.; Sato, Y.; Kawashima, M.; Furumichi, M.; Tanabe, M. KEGG as a reference resource for gene and protein annotation. Nucleic Acids Res. 2016, 44, D457-62, doi:10.1093/nar/gkv1070.

16. Altschul, S.F.; Madden, T.L.; Schäffer, A.A.; Zhang, J.; Zhang, Z.; Miller, W.; Lipman, D.J. Gapped BLAST and PSI-BLAST: A new generation of protein database search programs. Nucleic Acids Res. 1997, 25, 3389-3402, doi:10.1093/nar/25.17.3389. 
17. Boratyn, G.; Camacho, C.; Cooper, P.; Coulouris, G.; Fong, A.; Ma, N.; Madden, T.; Matten, W.; McGinnis, S.; Merezhuk, Y.; et al. BLAST: A more efficient report with usability improvements. Nucleic Acids Res. 2013, 41, W29-W33, doi:10.1093/nar/gkt282.

18. Kinsella, R.; Kähäri, A.; Haider, S.; Zamora, J.; Proctor, G.; Spudich, G.; Almeida-King, J.; Staines, D.; Derwent, P.; Kerhornou, A.; et al. Ensembl BioMarts: A hub for data retrieval across taxonomic space. Database (Oxf.) 2011, 2011, bar030, doi:10.1093/database/bar030.

19. Ruffier, M.; Kähäri, A.; Komorowska, M.; Keenan, S.; Laird, M.; Longden, I.; Proctor, G.; Searle, S.; Staines, D.; Taylor, K.; et al. Ensembl core software resources: Storage and programmatic access for DNA sequence and genome annotation. Database (Oxf.) 2017, 2017, bax020, doi:10.1093/database/bax020.

20. Yates, A.; Beal, K.; Keenan, S.; McLaren, W.; Pignatelli, M.; Ritchie, G.R.; Ruffier, M.; Taylor, K.; Vullo, A.; Flicek, P. The Ensembl REST API: Ensembl Data for Any Language. Bioinformatics 2015, 31, 143-145, doi:10.1093/bioinformatics/btu613.

21. Hung, J.H.; Weng, Z. Visualizing Genomic Annotations with the UCSC Genome Browser. Cold Spring Harb. Protoc. 2016, 2016, doi:10.1101/pdb.prot093062.

22. Haeussler, M.; Zweig, A.; Tyner, C.; Speir, M.; Rosenbloom, K.; Raney, B.; Lee, C.; Lee, B.; Hinrichs, A.; Gonzalez, J.; et al. The UCSC Genome Browser database: 2019 update. Nucleic Acids Res. 2019, 47, D853-D858, doi:10.1093/nar/gky1095.

23. Goecks, J.; Nekrutenko, A.; Taylor, J.; Team, G. Galaxy: A comprehensive approach for supporting accessible, reproducible, and transparent computational research in the life sciences. Genome Biol. 2010, 11, R86, doi:10.1186/gb-2010-11-8-r86.

24. Tyurin, A.; Kabardaeva, K.; Gra, O.; Mustafaev, O.; Sadovskaya, N.; Pavlenko, O.; Goldenkova-Pavlova, I. Efficient Expression of a heterologous gene in plants depends on the nucleotide composition of mRNA's 5'-region. Russ. J. Plant. Physiol. 2016, 63, 511-522, doi:10.1134/s1021443716030158.

25. Kozak, M. Circumstances and mechanisms of inhibition of translation by secondary structure in eucaryotic mRNAs. Mol. Cell. Biol. 1989, 9, 5134-5142, doi:10.1128/MCB.9.11.5134.

26. Ikemura, T. Codon usage and tRNA content in unicellular and multicellular organisms. Mol. Biol. Evol. 1985, 2, 13-34, doi:10.1093/oxfordjournals.molbev.a040335.

27. Plotkin, J.; Kudla, G. Synonymous but not the same: The causes and consequences of codon bias. Nat. Rev. Genet. 2011, 12, 32-42, doi:10.1038/nrg2899.

28. Quax, T.; Claassens, N.; Söll, D.; van der Oost, J. Codon Bias as a Means to Fine-Tune Gene Expression. Mol. Cell. 2015, 59, 149-161, doi:10.1016/j.molcel.2015.05.035.

29. Song, H.; Gao, H.; Liu, J.; Tian, P.; Nan, Z. Comprehensive analysis of correlations among codon usage bias, gene expression, and substitution rate in Arachis duranensis and Arachis ipaënsis orthologs. Sci. Rep. 2017, 7, 14853, doi:10.1038/s41598-017-13981-1.

30. Lingemann, M.; Liu, X.; Surman, S.; Liang, B.; Herbert, R.; Hackenberg, A.; Buchholz, U.; Collins, P.; Munir, S. Attenuated Human Parainfluenza Virus Type 1 Expressing Ebola Virus Glycoprotein GP Administered Intranasally Is Immunogenic in African Green Monkeys. J. Virol. 2017, 91, e02469-16, doi:10.1128/JVI.02469-16.

31. Zheng, Y.; Zhao, W.M.; Wang, H.; Zhou, Y.B.; Luan, Y.; Qi, M., Cheng, Y.Z.; Tang, W.; Liu, J.; Yu, H.; et al. Codon usage bias in Chlamydia trachomatis and the effect of codon modification in the MOMP gene on immune responses to vaccination. Biochem. Cell Biol. 2007, 85, 218-226, doi:10.1139/o06-211.

32. Picardi, E.; Pesole, G. Computational methods for ab initio and comparative gene finding. Methods Mol. Biol. 2010, 609, 269-284, doi:10.1007/978-1-60327-241-4_16.

33. König, S.; Romoth, L.; Gerischer, L.; Stanke, M. Simultaneous gene finding in multiple genomes. Bioinformatics 2016, 32, 3388-3395, doi:10.1093/bioinformatics/btw494.

34. Zhou, T.; Gu, W.; Ma, J.; Su,n X.; Lu, Z. Analysis of synonymous codon usage in H5N1 virus and other influenza A viruses. Biosystems 2005, 81, 77-86, doi:10.1016/j.biosystems.2005.03.002.

35. Kim, N.; Lim, S.; Chae, H.; Park, Y. Complete mitochondrial genome of the Amur hedgehog Erinaceus amurensis (Erinaceidae) and higher phylogeny of the family Erinaceidae. Genet. Mol. Res. 2017, 16, doi:10.4238/gmr16019300.

36. Kinkema, M.; Geijskes, J.; Delucca, P.; Palupe, A.; Shand, K.; Coleman, H.; Brinin, A.; Williams, B.; Sainz, M.; Dale, J. Improved molecular tools for sugar cane biotechnology. Plant Mol. Biol. 2014, 84, 497-508, doi:10.1007/s11103-013-0147-8. 
37. Chaney, J.; Clark, P. Roles for Synonymous Codon Usage in Protein Biogenesis. Annu. Rev. Biophys. 2015, 44, 143-166, doi:10.1146/annurev-biophys-060414-034333.

38. Tuller, T.; Carmi, A.; Vestsigian, K.; Navon, S.; Dorfan, Y.; Zaborske, J.; Pan, T.; Dahan, O.; Furman, I.; Pilpel, Y. An evolutionarily conserved mechanism for controlling the efficiency of protein translation. Cell 2010, 141, 344-354, doi:10.1016/j.cell.2010.03.031.

39. Whittle, C.; Extavour, C. Expression-Linked Patterns of Codon Usage, Amino Acid Frequency, and Protein Length in the Basally Branching Arthropod Parasteatoda tepidariorum. Genome Biol. Evol. 2016, 8, 2722-2736, doi:10.1093/gbe/evw068.

40. Tian, J.; Yan, Y.; Yue, Q.; Liu, X.; Chu, X.; Wu, N.; Fan, Y. Predicting synonymous codon usage and optimizing the heterologous gene for expression in E. coli. Sci. Rep. 2017, 7, 9926, doi:10.1038/s41598-017-10546-0.

41. Diament, A.; Pinter, R.; Tuller, T. Three-dimensional eukaryotic genomic organization is strongly correlated with codon usage expression and function. Nat. Commun. 2014, 5, 5876, doi:10.1038/ncomms6876.

Publisher's Note: MDPI stays neutral with regard to jurisdictional claims in published maps and institutional affiliations.

(C) 2020 by the authors. Licensee MDPI, Basel, Switzerland. This article is an open access article distributed under the terms and conditions of the Creative Commons Attribution (CC BY) license (http://creativecommons.org/licenses/by/4.0/). 